\title{
En guise de conclusion. Quelques jalons pour une nouvelle histoire des relations extérieures et de la diplomatie
}

\section{Christian Windler}

\author{
(2) OpenEdition \\ Journals \\ Édition électronique \\ URL : http://journals.openedition.org/edl/270 \\ DOI : $10.4000 /$ edl. 270 \\ ISSN : 2296-5084 \\ Éditeur \\ Université de Lausanne \\ Édition imprimée \\ Date de publication : 15 septembre 2010 \\ Pagination : 245-258 \\ ISBN : 978-2-940331-23-9 \\ ISSN : 0014-2026
}

Référence électronique

Christian Windler, «En guise de conclusion. Quelques jalons pour une nouvelle histoire des relations extérieures et de la diplomatie », Études de lettres [En ligne], 3 | 2010, mis en ligne le 15 septembre 2013, consulté le 19 décembre 2020. URL : http://journals.openedition.org/edl/270 ; DOI : https:// doi.org/10.4000/edl. 270 


\section{EN GUISE DE CONCLUSION \\ QUELQUES JALONS POUR UNE NOUVELLE HISTOIRE DES RELATIONS EXTÉRIEURES ET DE LA DIPLOMATIE}

Si, en 2002, l'auteur de cette contribution dressait un bilan plutôt négatif de la recherche, notamment francophone, dans le domaine de l'histoire des relations extérieures et de la diplomatie ${ }^{1}$, force est de constater aujourd'hui les transformations intervenues à cet égard. En effet, malgré la réticence de certains des principaux spécialistes à remettre en cause les problématiques et les méthodes d'analyse ${ }^{2}$, l'ouverture vers les sciences sociales et la prise en compte de l'apport des autres domaines des sciences historiques sont en train de renouveler l'histoire des relations extérieures $^{3}$. Une des voies de ce renouvellement (qui, à terme, devrait permettre de comprendre les pratiques socioculturelles du domaine des relations extérieures comme partie intégrante d'une histoire des faits de société) passe par l'accent mis sur le travail quotidien des intermédiaires - leurs pratiques de l'interaction et de la négociation, leurs expériences de l'altérité -, plutôt que sur les résultats des négociations ${ }^{4}$. On peut également signaler l'intérêt accru pour une histoire des intermédiaires

I. Ch. Windler, La diplomatie comme expérience de l'Autre, p. 27-29. L'auteur remercie Sandrine Picaud-Monnerat, Guillaume Poisson et Nadir Weber de la relecture soigneuse de ce texte.

2. L. Bély, L'art de la paix en Europe.

3. Voir les contributions dans H. von Thiessen und Ch. Windler (Hrsg.), Akteure $\operatorname{der}$ Außenbeziehungen.

4. Voir à ce propos S. Andretta et al. (éds), Paroles de négociateurs, p. 1-26; J.-C. Waquet, "Verhandeln in der Frühen Neuzeit»; id., François de Callières. 
subalternes ${ }^{5}$. Ces derniers, à cause de leur relatif effacement, n'avaient pas trouvé l'attention qu'ils auraient méritée par leur participation à la conduite quotidienne des affaires. De même, notamment grâce à l'intégration d'une perspective "gender", les intermédiaires sans emploi formel voient leur rôle mis en lumière ${ }^{6}$.

Le titre du colloque - "Le diplomate en question" - de même que l'accent mis sur les relations avec le Corps helvétique au sens large, incluant, outre les XIII cantons, Genève ou les Grisons, ont pu être perçus comme une prise de distance à l'égard d'une histoire de la diplomatie centrée sur les diplomates de rang élevé, compris comme des acteurs de relations internationales au sens des $\mathrm{XIX}^{\mathrm{e}}$ et $\mathrm{XX}^{\mathrm{e}}$ siècles et exclusivement consacrés au service d'un Etat. D’une part, la plupart des représentants diplomatiques dans les cantons étaient d'un rang plutôt modeste, d'autre part, le Corps helvétique et ses différentes composantes étaient très loin de correspondre à un Idealtyp étatique.

Si l'on s'en tenait seulement à l'étymologie, une histoire de la diplomatie du Bas Moyen Age ou de l'époque moderne serait une histoire sans objet: en effet, ce n'est qu'à la fin du XVIII' siècle que les acceptions actuelles des termes "diplomatique», «diplomate» et «diplomatie» (et de leurs équivalents dans d'autres langues européennes, comme le souligne Antonio Trampus pour l'italien) s'imposent. Jusque-là, l'adjectif et le substantif "diplomatique" se référaient à ce qui est relatif aux "diplômes", civils ou ecclésiastiques, ayant trait, de façon indifférente, aux relations extérieures et à des questions internes aux entités politiques.

Même s'il ne doit pas empêcher l'historien d'utiliser les termes dans un but analytique pour des époques plus lointaines, ce constat de l'étymologie ne peut lui être indifférent. Il est à mettre en rapport avec les transformations intervenues autour de 1800, qui ont favorisé l'essor d'un système de relations proprement «internationales" (c'est-à-dire de relations entre des communautés s'imaginant comme des Nations et organisées dans un cadre étatique), dont les intermédiaires connurent une certaine professionnalisation ${ }^{7}$. En s'inspirant de la terminologie de Reinhart Koselleck, on pourrait décrire le tournant des XVIII et

5. Voir, par exemple, J. Ulbert et G. Le Bouëdec (éds), La fonction consulaire à l'époque moderne.

6. Voir, à titre d'exemple, E. K. Dade, Madame de Pompadour.

7. M. Belissa, Repenser l'ordre européen 1795-1802. 
XIX ${ }^{\mathrm{e}}$ siècles comme une Sattelzeit diplomatique. Dans le contexte politique de la Restauration, le Congrès de Vienne aurait consacré les nouvelles règles d'interaction internationale issues des révolutions de la fin du XVIII e siècle, notamment l'égalité juridique d'Etats souverains et le statut correspondant de leurs représentants diplomatiques. Ces transformations furent sans aucun doute beaucoup plus profondes que celles que les sciences politiques (plutôt que la recherche historique) ont tendance à associer aux traités de Westphalie, laissant à entendre que les relations extérieures, après 1648, auraient été dominées plus ou moins exclusivement par des logiques étatiques, caractérisées par l'interaction d'Etats souverains selon les règles du droit des Gens ${ }^{8}$.

Dans cette perspective, il convient de retenir que la conduite de relations interétatiques par l'entremise de diplomates professionnels menant une véritable "carrière diplomatique» et se consacrant de façon plus ou moins exclusive à ces tâches est un fait récent qui échappe, dans une large mesure, aux périodes couvertes par le colloque.

Dans la France du XVIII e siècle, la constitution de corps d'intermédiaires professionnalisés est généralement limitée à des domaines où la nécessité de connaissances spécifiques était particulièrement nette. Ainsi, à partir de la fin du XVII e siècle, les ordonnances de Marine ont tendance à définir les contours de véritables corps spécialisés de consuls et de drogmans du Levant et de "Barbarie», distinct des consuls de la Chrétienté 9 . Lordonnance de 1781 définit les normes qui devaient régir dorénavant le recrutement et la carrière des consuls et des drogmans, dont il est dit qu'ils appartenaient à une "administration» et dont le ministère était réservé explicitement "pour le service et pour l'intérêt public». Dès 1776, une ordonnance provisoire avait introduit une stricte hiérarchie des postes consulaires; par ailleurs, l'avancement d'une catégorie de poste à une autre devait désormais être régi par l'ancienneté et le mérite. La pension de retraite, échelonnée selon les années de service,

8. Voir par exemple, E. Krippendorff, «Die Erfindung der Außenpolitik»; E. Luard, The Balance of Power. Pour une critique du concept de «système westphalien", cf. B. Teschke, The Myth of 1648.

9. Voir Ch. Windler, La diplomatie comme expérience de l'Autre, notamment p. 37 sq. Ces observations sont à rapprocher des remarques d'Antonio Trampus dans le présent volume qui insiste, à juste titre, sur la nécessité de tenir compte du rôle des consuls dans la promotion des activités économiques et des relations politiques. 
apparaissait comme le prix du dévouement professionnel de toute une vie.

Ces normes, mais aussi les pratiques qu'elles inspirent, vont bien audelà de ce que l'on peut trouver dans les pays chrétiens, même chez des intermédiaires placés aux échelons inférieurs, par exemple les résidents français à Genève (étudiés par Fabrice Brandli). Les personnages qui font l'objet des contributions réunies dans le présent volume ont suivi, plutôt qu'une "carrière diplomatique», un cursus honorum dans lequel les fonctions d'intermédiaire diplomatique ont occupé une place plus ou moins considérable, mais jamais exclusive. A côté de cette insertion dans un cursus honorum menant vers des honneurs plus considérables, on trouve même le cas, exceptionnel pour la diplomatie espagnole du XVII ${ }^{\mathrm{e}}$ siècle et sans doute motivé par les exigences particulières du service dans les cantons, d'un quasi-monopole familial sur une représentation diplomatique pendant plus d'un siècle. En effet, les Casati, analysés par Andreas Behr, suivaient une logique patrimoniale plutôt que celle d'un cursus honorum; les fonctions diplomatiques définissaient l'état de la famille sur plusieurs générations, d'une façon analogue à l'achat et à la transmission héréditaire d'un office.

Plus que par les fonctions exclusivement diplomatiques, le statut social des intermédiaires se définissait par les services divers qu'ils rendaient à un prince ou à une communauté et, aussi, par leur intégration à des réseaux de parenté et de clientèle. Même au XVIII ${ }^{\mathrm{e}}$ siècle, que ce fût en France, en Grande-Bretagne ou dans l'Empire, la position sociale du diplomate n'était pas définie par les appointements rémunérant ses activités en ce domaine. Le bénéfice que promettaient les ambassades était plus social qu'immédiatement matériel. Le fait de représenter son prince augmentait, avant tout, le capital social de l'ambassadeur et de sa famille. C'était également le cas des intermédiaires, en général d'un rang plus modeste, qui représentaient les cours étrangères dans le Corps helvétique. Certes, ils recevaient des appointements et étaient défrayés des dépenses faites pour le service, mais ces paiements n'expliquent pas à eux seuls l'intérêt qu'ils pouvaient avoir à accepter, voire à rechercher, des missions diplomatiques, comme il ressort notamment des contributions d'Aureliano Martini, d'Antonio Trampus, de Guillaume Poisson et d'Andreas Behr. 
Ce constat ne prend tout son sens que dans le contexte de la remise en question, depuis les années 1980, de la notion d'absolutisme dans l'analyse des rapports de domination internes. Même si on ne partage pas les conclusions les plus radicales d'un Nicholas Henshall ${ }^{10}$, le débat que celles-ci ont contribué à alimenter a posé des jalons tout aussi incontournables pour l'histoire des relations extérieures que pour celle des rapports internes de domination ${ }^{11}$. Ainsi, Helmut Koenigsberger et John Elliott ont souligné les limites du pouvoir princier résultant du caractère composé des monarchies de l'époque moderne ${ }^{12}$. Dans le même contexte scientifique, ont été mises en lumière les limites de la bureaucratisation dans l'exercice du pouvoir, le poids des logiques clientélaires dans le recrutement des officiers, et le rôle primordial des relations de type personnel pour l'intégration des périphéries ${ }^{13}$. La «microhistoire» a proposé de nouveaux modèles d'interprétation des processus de formation des structures étatiques, en centrant l'attention sur des interactions que des acteurs subalternes étaient souvent capables d'orienter dans des directions qui leur convenaient ${ }^{14}$.

Sur ce fond de déconstruction du paradigme absolutiste, il convient de ne pas considérer les intermédiaires diplomatiques de l'époque moderne dans leur seul rôle d'exécuteurs de la politique extérieure de leurs gouvernements. Cette considération a amené Hillard von Thiessen à proposer, pour la période comprise entre les mutations de la fin du Moyen Age et la Sattelzeit autour de 1800, le concept d'une «diplomatie de type ancien", définie notamment par les traits suivants: à l'instar des

Io. N. Henshall, The Myth of Absolutism.

II. Sur ce débat, voir R. G. Asch und H. Duchhardt (Hrsg.), Der Absolutismus - ein Mythos?; L. Schilling (Hrsg.), Der Absolutismus, ein unersetzliches Forschungskonzept?

I2. J. H. Elliott, "A Europe of composite monarchies»; H. G. Koenigsberger, «Dominum Regale oder Dominium Politicum et Regale».

I3. Dans le contexte germanophone, Wolfgang Reinhard a contribué de façon décisive à l'introduction de l'analyse des réseaux dans les sciences historiques. Voir W. Reinhard, Freunde und Kreaturen. De manière parallèle, des historiens anglais et américains l'ont introduite dans l'étude de la monarchie française. Voir Sh. Kettering, Patrons, Brokers and Clients in seventeenth Century France. Cf. B. Emich, Territoriale Integration in der Frühen Neuzeit.

I4. Parmi les travaux plus anciens, voir l'œuvre particulièrement influente de Giovanni Levi: G. Levi, L'eredità inmateriale. Pour un bon aperçu d'approches plus récentes, voir W. Blockmans, A. Holenstein and J. Mathieu (eds), Empowering Interactions. 
rapports de domination interne, les relations extérieures se présentaient sous forme de réseaux personnels. Les intermédiaires diplomatiques se caractérisaient par la diversité de leurs rôles sociaux, qui résultaient à leur tour de la diversité des réseaux dans lesquels ils étaient intégrés. Dans des relations sociales qui fonctionnaient selon les normes d'échanges de dons et de contre dons, ils jouaient non seulement le rôle de serviteurs d'un prince ou d'une communauté, mais aussi, par exemple, celui de membres de réseaux de parenté ou de clientèle, auxquels ils devaient des prestations tout autant qu'au prince ou à la communauté qui les employait. La diplomatie "de type ancien» se caractérisait donc par la pluralité des rôles et des normes, plutôt que par le service exclusif rendu à un prince ou à une communauté. Dans des sociétés caractérisées par la pluralité des systèmes normatifs, la primauté de leurs obligations n'était pas fixée d'avance, mais dépendait du contexte et du point de vue respectif des acteurs $^{15}$.

S’il leur arrivait de réclamer la primauté des obligations à leur égard, les princes ou les communautés misaient tout autant sur la diversité des réseaux de leurs intermédiaires diplomatiques et, partant, sur la multiplicité des savoirs dont ils pouvaient tirer profit à travers ces différents réseaux. Ainsi, comme le montre le portrait qu'Eva Pibiri dresse de deux envoyés qui jouissaient d'un double ancrage, savoyard et bernois, le duc de Savoie mettait à profit les réseaux sociaux et les compétences linguistiques de personnages habitués à passer les frontières - le patricien bernois Konrad von Scharnachthal avait bénéficié, en la matière, de son éducation en tant que page à la cour d'Amédée VIII. En envoyant Antonio Riboldi da Besana à la diète confédérée, le duc de Milan Francesco Sforza recourait, à l'instar d'Amédée VIII de Savoie et de son fils, aux services d'un officier à qui l'activité dans le voisinage des Suisses avait donné une familiarité personnelle jugée utile pour une mission difficile.

Dans la perspective de Milan et de la cour de Madrid, au XVII e siècle encore, les avantages du maintien d'une fonction au sein d'une même famille semblaient supérieurs à ses inconvénients potentiels. Ainsi, les rois d'Espagne permirent à la famille Casati d'exercer, pendant plus d'un siècle, un quasi-monopole sur la représentation milanaise dans

15. H. von Thiessen, "Diplomatie vom type ancien». Voir du même auteur, Grenzüberschreitende Patronage und Diplomatie vom type ancien. 
les cantons; la permanence sur les mêmes lieux et l'intégration dans la société d'accueil, allant jusqu'à l'entrée d'Alfonso Casati, en 1680, dans le patriciat de Lucerne, impliquaient une gestion de cette représentation par et pour la famille dont les relations, ainsi que les connaissances accumulées au cours du temps, étaient mises parallèlement au service de la monarchie hispanique. La contribution d'Andreas Behr fait également voir comment, au XVII e siècle, les relations extérieures d'une monarchie composée échappaient encore à une définition claire de la souveraineté dans les termes de Jean Bodin. Les Casati étaient-ils des envoyés de Philippe II et de ses successeurs en tant que rois d'Espagne ou en tant que ducs de Milan, ou des deux à la fois? Quelles étaient les voies de leur nomination et leur titre précis? Si on ajoutait au cas milanais celui du Comté de Bourgogne, on verrait encore mieux comment, dans certains contextes, les monarchies composées laissaient aux acteurs subalternes, même dans leurs relations extérieures, une marge de manœuvre considérable, leur permettant à l'occasion d'instrumentaliser la politique des princes ${ }^{16}$.

Dans le cas de la représentation française à Soleure, objet de la contribution de Guillaume Poisson, si les ambassadeurs changeaient régulièrement, les secrétaires-interprètes, recrutés localement, bien qu'issus en partie de familles d'origine française ayant acquis le droit de bourgeoisie à Soleure, savaient se rendre indispensables par leurs relations étroites avec le patriciat de la ville. Ainsi, pendant plus de deux siècles, la famille Vigier transmit à sa lignée l'héritage de ses bons services rendus au roi de France.

Les contributions consacrées aux messagers et ambassadeurs des princes et des villes de la fin du Moyen Age, c'est-à-dire les textes de Jean-Marie Moeglin, Eva Pibiri, Klara Hübner, Aureliano Martini et Anne-Brigitte Spitzbarth, mettent certes en lumière l'importance de ce premier moment d'affirmation d'une diplomatie fondée sur des ambassadeurs dotés d'un "véritable statut", impliquant «une hiérarchie et une différenciation fonctionnelle entre les différents types de représentants». Ceux-ci ont trouvé toute leur importance dans un contexte qui a vu la disparition progressive des rencontres personnelles des princes. Les envoyés ad hoc qui préparaient les rencontres des princes furent

I6. Sur les marges de manœuvre des acteurs subalternes dans ce contexte, voir Ch. Windler, «Außenbeziehungen vor Ort». 
substitués par des ambassadeurs capables d'assurer la représentation de leur maître absent, d' "en incarner la grandeur et la majesté» (JeanMarie Moeglin). A la même époque s'accentua le caractère technique des missions diplomatiques, ce qui rendit nécessaire la présence de juristes dans les négociations. Pourtant, dans le cas des cantons suisses, l'installation des premiers ambassadeurs résidents n'intervint qu'au cours du $\mathrm{XVI}^{\mathrm{e}}$ siècle.

Si l'on tient compte de l'étude de Christopher Storrs sur la diplomatie britannique, qui montre, en plein XVIII e siècle encore, les limites de l'organisation administrative, il convient de ne pas surestimer le degré de spécialisation des envoyés de la fin du Moyen Age et ce, en dépit d'un début de concentration des tâches, nettement perceptible, entre les mains d'un petit nombre de personnes. «Sens", "loyauté", "prudence», "discrétion" et "prudhommie» devaient caractériser les ambassadeurs bourguignons; or, ces qualités n'étaient pas spécifiques d'un négociateur. Anne-Brigitte Spitzbarth en conclut à juste titre que le «savoir être» était le critère primordial du choix du négociateur par le prince, la compétence juridique et procédurière des juristes de formation n'étant certes pas négligeable, mais occupant une place secondaire. En guise de conclusion, dans des termes proches de ceux de Jean-Marie Moeglin, elle fait remarquer que «le chemin vers une réelle professionnalisation des ambassadeurs restait encore long à parcourir ».

Laccent mis sur les cantons suisses soulève la question des interactions entre différentes cultures politiques: la plupart des acteurs présentés dans les contributions venaient de contrées marquées par des cultures monarchiques; or, ils agirent dans des communautés qui, d'une appartenance au Saint Empire assortie d'amples privilèges, évoluèrent à partir de la seconde moitié du XVII ${ }^{\mathrm{e}}$ siècle, selon un processus pourtant lent et inégal selon les lieux, vers l'affirmation d'une souveraineté républicaine ${ }^{17}$.

Les relations des pouvoirs princiers avec les cantons constituèrent donc, à différents égards, des "expériences de l'Autre ${ }^{18}$. A juste titre, Fabrice Brandli souligne la nécessité de "penser la diplomatie comme une pratique sociale de l'altérité que déterminent les normes culturelles antagonistes et mouvantes des acteurs impliqués» et qui "participe dans un

I7. Sur le langage politique dans le Corps helvétique, voir Th. Maissen, Die Geburt der Republic.

18. Ch. Windler, «Diplomatie als Erfahrung fremder politischer Kulturen». 
jeu de chassé-croisé à l'élaboration [...] de l'identité des protagonistes". Dans sa contribution, il se propose de mesurer les rapports entre les profils des intermédiaires diplomatiques français à Genève et la culture politique qui encadrait leurs pratiques et leurs représentations; l'auteur insiste sur la perception du républicanisme genevois comme contraire à une rationalité politique monarchique jugée supérieure, et l'illustre plus particulièrement par l'analyse de l'amalgame entre les représentations du républicain réformé et celles du juif, l'un et l'autre étant assimilés à des financiers agioteurs et à des marchands contrebandiers.

Les différences culturelles, religieuses, sociales et politiques entre les milieux d'origine des interlocuteurs devaient naturellement susciter des difficultés de communication plus ou moins grandes. Aureliano Martini met en valeur la capacité d'Antonio Riboldi da Besana, "orateur» du duc de Milan, à comprendre le fonctionnement particulier du système des ligues suisses et à conduire les négociations non seulement au sein de la diète, mais encore dans les cantons, aussi bien au niveau formel des institutions qu'à celui plus personnel des contacts avec les notables susceptibles d'influer sur les décisions. On peut également deviner l'importance de ces mêmes compétences derrière la permanence de quelques familles dans les fonctions de secrétaires-interprètes du roi de France à Soleure, objet de la contribution de Guillaume Poisson.

Les interactions quotidiennes ne supposaient pas seulement la connaissance des institutions et la familiarité avec les personnes, mais encore un savoir précis sur les gestes qui, par leur valeur symbolique, donnaient une signification déterminée aux relations. En effet, comme l'ont récemment rappelé les recherches sur la communication symbolique conduites sous la direction de Barbara Stollberg-Rilinger et les travaux de Rudolf Schlögl ${ }^{19}$, dans les sociétés européennes d'Ancien Régime les ordres sociaux étaient produits et reproduits in actu entre les personnes présentes. La création des normes dans les interactions face à face conduisait les acteurs à considérer les échanges du quotidien comme autant d'occasions permettant d'affirmer l'ordre des relations réciproques et exigeant donc l'investissement de tous les interlocuteurs. L'inégalité

19. B. Stollberg-Rilinger, "Symbolische Kommunikation in der Vormoderne"; M. Füssel und Th. Weller (Hrsg.), Ordnung und Distinktion; R. Schlögl (Hrsg.), Interaktion und Herrschaft, p. 9-62; R. Schlögl, B. Giesen und J. Osterhammel (Hrsg.), Die Wirklichkeit der Symbole. 
qui caractérisait les sociétés d'Ancien Régime se retrouvait dans les relations extérieures entre les entités politiques. Plutôt que par l'égalité d'Etats souverains, ces relations étaient caractérisées par un ordre hiérarchique dans lequel les princes et les communautés s'inséraient de façon agonistique, notamment à travers le cérémonial. Le rôle essentiel que des questions comme les ordres de préséance ou les titulatures jouaient dans la constitution des hiérarchies sociales explique les nombreux conflits qu'elles suscitaient ${ }^{20}$. Sans doute serait-il intéressant de voir comment les pratiques de l'interaction diplomatique dans le Corps helvétique changèrent au fur et à mesure des transformations des cultures politiques des cantons, notamment à partir de la seconde moitié du XVII $\mathrm{e}^{\mathrm{e}}$ siècle.

Par ailleurs, il convient de se demander si les exigences particulières du milieu suisse eurent une influence lors de la sélection des intermédiaires destinés à y être envoyés. Certaines contributions semblent permettre de répondre par l'affirmative. Cependant, comme l'illustrent de manière saisissante les Casati ou les secrétaires-interprètes du roi de France à Soleure, spécialisation n'impliquait pas nécessairement professionnalisation, voire bureaucratisation. Ces réponses au défi des rapports avec les cantons correspondaient à la «diversité des états intégralement sociaux» qui caractérisait les cultures politiques d'Ancien Régime ${ }^{21}$. Les solutions se trouvaient à l'opposé des rationalités qui, dans la France du XVIII siècle, guideront la constitution d'une "carrière" des consuls du Levant et de «Barbarie». Il serait cependant trop tôt pour conclure que, dans les contextes donnés, elles étaient moins efficaces.

\section{Christian WindLER Université de Berne}

20. Voir, en particulier A. Krischer, Reichsstädte in der Fürstengesellschaft. Sur des contextes mettant en rapport des acteurs européens et non européens, voir Ch. Windler, La diplomatie comme expérience de l'Autre, en particulier p. 405-484.

2I. B. Clavero, Antidora, p. 195. 


\section{BIBLIOGRAPHIE}

Andretta, Stefano, Péquignot, Stéphane, Schaub, Marie-Karine, Waquet, Jean-Claude et Windler, Christian (éds), Paroles de négociateurs. L'entretien dans la pratique diplomatique de la fin du Moyen âge à la fin du XIX ${ }^{e}$ siècle, Rome, Ecole française de Rome, 2010.

Asch, Ronald G. und Duchrardt, Heinz (Hrsg.), Der Absolutismus ein Mythos? Strukturwandel monarchischer Herrschaft in West- und Mitteleuropa (ca. 1550-1700), Köln, Böhlau Verlag, 1996.

Belissa, Marc, Repenser l'ordre européen 1795-1802. De la société des rois aux droits des nations, Paris, Editions Kimé, 2006.

BéLy, Lucien, L'art de la paix en Europe. Naissance de la diplomatie moderne, XVI ${ }^{e}$-XVIII e siècle, Paris, PUF, 2007.

Blockmans, Wim, Holenstein, André and Mathieu, Jon (eds), Empowering Interactions. Political Cultures and the Emergence of the State in Europe 1300-1900, Farnham, Ashgate, 2009.

Clavero, Bartolomé, Antidora. Antropología católica de la economía moderna, Milano, Giuffré Editore, 1991.

Dade, Eva Kathrin, Madame de Pompadour. Die Mätresse und die Diplomatie, Köln, Böhlau Verlag, 2010.

Elliott, John Huxtable, "A Europe of composite monarchies", Past and Present, 137 (1992), p. 48-71.

Емісн, Birgit, Territoriale Integration in der Frühen Neuzeit. Ferrara und der Kirchenstaat, Köln, Böhlau Verlag, 2005.

Füssel, Marian und Weller, Thomas (Hrsg.), Ordnung und Distinktion. Praktiken sozialer Repräsentation in der ständischen Gesellschaft, Münster, Rhema, 2005.

Henshall, Nicholas, The Myth of Absolutism. Change and Continuity in Early Modern European Monarchy, London, Longman, 1992.

Kettering, Sharon, Patrons, Brokers and Clients in Seventeenth Century France, New York/Oxford, Oxford U.P., 1986. 
Koenigsberger, Helmut Georg, "Dominum Regale oder Dominium Politicum et Regale. Monarchies and Parliaments in Early Modern Europe", in Politicians and virtuosi. Essays in Early Modern History, London, The Hambledon Press, 1986, p. 1-25.

KrippendorfF, Ekkehart, "Die Erfindung der Außenpolitik», in

Strukturwandel Internationaler Beziehungen. Zum Verhältnis von

Staat und Internationalem System seit dem Westfälischen Frieden, hrsg. von Jens Siegelberg und Klaus Schlichte, Wiesbaden, Westdeutscher Verlag, 2000, p. 61-73.

Krischer, André, Reichsstädte in der Fürstengesellschaft. Politischer Zeichengebrauch in der Frühen Neuzeit, Darmstadt, Wissenschaftliche Buchgesellschaft, 2006.

Levi, Giovanni, L'eredità inmateriale. Carriera di un esorcista nel Piemonte del Seicento, Torino, L. Einaudi, 1985.

Luard, Evan, The Balance of Power. The System of International Relations, 1648-1815, Basingstoke, Macmillan, 1992.

Maissen, Thomas, Die Geburt der Republic. Staatsverständnis und Repräsentation in der frühneuzeitlichen Eidgenossenschaft, Göttingen, Vandenhoeck \& Ruprecht Verlag, 2006.

Reinhard, Wolfgang, Freunde und Kreaturen. „Verflechtung“ als Konzept zur Erforschung historischer Führungsgruppen. Römische Oligarchie um 1600, München, Ernst Vögel, 1979.

Schilling, Lothar (Hrsg.), Der Absolutismus, ein unersetzliches Forschungskonzept? Eine deutsch-französische Bilanz / L'absolutisme, un concept irremplaçable? Une mise au point franco-allemande, München, R. Oldenbourg Verlag, 2008.

Schlögl, Rudolf (Hrsg.), Interaktion und Herrschaft. Die Politik der frühneuzeitlichen Stadt, Konstanz, UVK, 2004.

Schlögl, Rudolf, Giesen, Bernhard und Osterhammel, Jürgen (Hrsg.), Die Wirklichkeit der Symbole. Grundlagen der Kommunikation in historischen und gegenwärtigen Gesellschaften, Konstanz, UVK, 2004.

Stollberg-Rilinger, Barbara, "Symbolische Kommunikation in der Vormoderne. Begriffe - Thesen - Forschungsperspektiven", Zeitschrift für historische Forschung, 31 (2004), p. 489-527.

Teschke, Benno, The Myth of 1648. Class, Geopolitics, and the Making of Modern International Relations, London/New York, Verso, 2003. 
Thiessen, Hillard von, «Diplomatie vom type ancien: Überlegungen zu einem Idealtypus des frühneuzeitlichen Gesandtschaftswesens", in Akteure der Außenbeziehungen. Netzwerke und Interkulturalität im historischen Wandel, hrsg. von Hillard von Thiessen und Christian Windler, Cologne, Böhlau Verlag, 2010, p. 471-503.

—, Grenzüberschreitende Patronage und Diplomatie vom type ancien. Die spanisch-römischen Beziehungen im Pontifikat Pauls V. (1605-1621) in akteurzentrierter Perspektive, Epfendorf, Bibliotheca Academica Verlag, 2010.

Thiessen, Hillard von und Windler, Christian (Hrsg.), Akteure der Außenbeziehungen. Netzwerke und Interkulturalität im historischen Wandel, Köln, Böhlau Verlag, 2010.

Ulbert, Jörg et Le BouëDec, Gérard (éds), La fonction consulaire à l'époque moderne. L'affirmation d'une institution économique et politique (1500-1700), Rennes, PUR, 2006.

Waquet, Jean-Claude, François de Callières. L'art de négocier en France sous Louis XIV, Paris, ENS, 2005.

—, "Verhandeln in der Frühen Neuzeit. Vom Orator zum Diplomaten», in Akteure der Außenbeziehungen. Netzzwerke und Interkulturalität im historischen Wandel, hrsg. von Hillard von Thiessen und Christian Windler, Köln, Böhlau Verlag, 2010, p. 113-131.

Windler, Christian, La diplomatie comme expérience de l'Autre. Consuls français au Maghreb (1700-1840), Genève, Droz, 2002.

—, "Außenbeziehungen vor Ort. Zwischen "großer Strategie” und Privileg», Historische Zeitschrift, 281 (2005), p. 593-619.

—, "Diplomatie als Erfahrung fremder politischer Kulturen: Gesandte von Monarchen in den eidgenössischen Orten (16. und 17. Jahrhundert)", in Geschichte und Gesellschaft. Zeitschrift für Historische Sozialwissenschaft, 32 (2006), p. 5-44. 
